\title{
Tangence
}

\section{Raymond Devos : artiste, humoriste et poète}

\section{Michèle Nevert}

Numéro 53, décembre 1996

L’humour de la poésie

URI : https://id.erudit.org/iderudit/025929ar

DOI : https://doi.org/10.7202/025929ar

Aller au sommaire du numéro

Éditeur(s)

Tangence

ISSN

0226-9554 (imprimé)

1710-0305 (numérique)

Découvrir la revue

Citer cet article

Nevert, M. (1996). Raymond Devos : artiste, humoriste et poète. Tangence, (53), 102-119. https://doi.org/10.7202/025929ar d'utilisation que vous pouvez consulter en ligne.

https://apropos.erudit.org/fr/usagers/politique-dutilisation/ 


\section{Raymond Devos : artiste, humoriste et poète Michèle Nevert}

L'habitude nous conduit depuis longtemps à inclure Raymond Devos dans la grande famille des clowns, inaugurée, pour les uns, par les bouffons et les mimes de l'Antiquité, pour d'autres, par les fous des rois de l'âge féodal ou les baladins du théâtre. Il est vrai que lorsqu'il fait devant nous l'exercice de la plupart des disciplines des fêtes foraines - équilibre, sauts périlleux, jonglerie, marche sur un fil, mime - il s'apparente, de fait, à l'homme de cirque. Sans compter la pratique systématique de nombreux instruments de musique (clarinette, piano, harpe, guitare, concertina, vibraphone, etc), l'exploitation et la transformation d'objets divers en sources sonores (bouteilles, verres, grelots) ou encore la création de nouveaux instruments (clarinette molle, cor de chasse qui se déroule, trombone à trois coulisses) depuis toujours dans ses spectacles. Pour autant, Devos s'éloigne aussi du clown, par l'absence de certains de ses traits spécifiques (tenue vestimentaire et maquillage particuliers) et par la présence d'éléments qui, en revanche, lui appartiennent en propre, tels ses jeux de langage. Du coup, et à première vue, on ne sait guère où le classer, ce qui pourrait déranger une grande partie de la critique universitaire, habile à délimiter des genres et favorable à leur maintien.

\section{Raymond Devos, l'artiste : un miroir}

Cette adhésion totale au bouffon d'antan, ce mode d'être de l'ancien bateleur médiéval nous force à nous interroger sur le type de fonction bien singulière que Devos assume dans la société. Car il est manifeste que les préoccupations sociales ne constituent pas, loin de là, l'essentiel de son cuvre ${ }^{1}$. Par comparaison avec d'autres qui les affectionnent particulièrement, on est frappé par la rareté des textes à teneur sociale et politique et par l'absence volontaire de références précises à l'intérieur

1 Raymond Devos, Matière à rire, Paris, Olivier Orban, 1991, 542 p. Dorénavant, les références à cet ouvrage seront identifiées par le sigle $M R$, suivi du folio. 
même de ces sketches, lors de la représentation. En vérité, Devos les met "en scène" dans le but d'affirmer son indifférence et de dénoncer ce qu'il faut comprendre, à ses yeux, comme la vanité de telles préoccupations. Parler pour ne rien dire en fournit une belle illustration: "Parlons de la situation [...] sans préciser laquelle! [...] je vais faire brièvement l'historique de la situation, quelle qu'elle soit!» ( $M R$, p. 273) Prouesse de ce monologue qui donne sur le mode typique de la langue de bois (toujours associée au discours politique) un texte cependant parfaitement significatif. Mais l'exercice de style présente un second intérêt, puisque en l'absence d'allusions particulières, le texte demeure toujours d'actualité malgré le changement de pouvoir en France depuis sa création, et conservera, on le présume, cette actualité, en dépit de toute variation possible du contexte politique: "Je vous signale entre parenthèses que si le gouvernement actuel n'est pas capable d'assumer la catastrophe, il est possible que l'opposition s'en empare!" ( $M R$, p. 274) Dans la même veine, on trouve à l'intérieur d'Entre parenthèses l'exemple suivant: "Vous savez ce qui est arrivé au ministre de... tsss... Ah! (Il cherche le nom.) Mais si! Celui qui promet... [...] C'est comme ce leader de l'opposition! Comment s'appelle-t-il?... Mais si !" ( $M R$, p. 315) “Il promet aussi!" ajoute-t-il parfois en spectacle.

Parmi les problèmes sociaux contemporains, et outre la faim et la misère, l'artiste semble davantage bouleversé par le racisme (Xénopbobie, MR, p. 300; En aparté, MR, p. 316; Racisme, $M R$, p. 317; Au lieu de se battre, $M R$, p. 340): "J'ai un ami qui est xénophobe. C'est-à-dire qu'il ne peut pas supporter les étrangers! [...] Il déteste à tel point les étrangers que lorsqu'il va dans leur pays, il ne peut pas se supporter!" (MR, p. 300); "- S'il a dit: Tiens! Voilà Truc!, c'est parce qu'il ne se souvenait pas du nom du Turc!» $(M R$, p. 316). Derrière l'ironie, le tour de passe-passe verbal et la lecture politique personnelle de l'humoriste ${ }^{2}$, l'artiste indique sa prédilection pour le jeu sur le signifiant, sur le côté sonore des mots, et affirme sa fonction intrinsèque de créateur de chimères pour les spectateurs et les lecteurs. C'est pourquoi, à l'inverse d'autres humoristes qui font de la situation politique et

2 Il faut se remémorer, à ce propos, sa présentation du concertina : "C'est l'instrument de l'alternance par excellence. Quand vous appuyez à gauche, ça souffle à droite et quand vous appuyez à droite, ça siffle à gauche. Et, à l'intérieur... c'est du vent!n 
104

des problèmes économiques l'objet de leurs textes, Raymond Devos ne se présente pas comme un miroir social. Son univers chimérique, sa façon d'utiliser le langage, l'absurdité et l'étrangeté des situations dans lesquelles il se montre, l'imposent comme une sorte de miroir de notre inconscient.

\section{Raymond Devos, l'humoriste : le maître des mots}

"Magicien des mots", "Prince de l'absurde", "Amant privilégié du langage", il n'est pas d'expressions utilisées pour nommer cet artiste qui ne désignent en même temps le rapport singulier qu'il entretient avec la langue. De fait, le cadre de ses textes est d'abord fourni par une exploitation magistrale des jeux langagiers: "Chaque fois que votre femme fait une fugue... vous, vous en écrivez une! " (MR, p. 518); "Comme j'avais entendu dire: à quand les vacances? [...] Je me dis: Bon!... je vais aller à Caen." (MR, p. 393). Lorsqu'il ne s'appuie pas sur les différents sens d'un même mot, Devos utilise fréquemment le phénomène de l'homophonie de plusieurs termes. Autrement dit, il s'empare des "doubles phoniques "que recèle la langue pour alimenter ses jeux d'écriture: "Je me vois devant moi $[$...] jugez de mon émoi" (MR, p. 188); "La penderie avait un double fond et tous les doubles [...] étaient au fond !" ( $M R$, p. 190). Ces doubles phoniques sont à l'origine des jeux verbaux sans doute les plus spectaculaires de Raymond Devos. Que l'on songe seulement à "l'ouie de l'oie de Louis a oui. Ah oui?" (MR, p. 82) dans Oui dire; ou encore dans Le savoir choir à "Chois comme ce mouchoir de soie... mon chou! Chois léger!" ( $M R$, p. 143)... Pour leur part, les "dédoublements sémantiques", ont engendré plusieurs formules percutantes qu'il est difficile ici de passer sous silence: "Dieu que ce que j'ois est triste!n ( $M R$, p. 81); "Se coucher tard... nuit!" ( $M R$, p. 310); "Le flux et le reflux me font marée!. ( $M R$, p. 271); "Je suis devenu un peigne [...] Parce que c'est la première chose qui me soit passée par la tête!॰ $(M R$, p. 161); "si je reste quelques jours sans la perdre... elle me manque!" ( $M R$, p. 178$)$; "[si] le Bon Dieu n'avait jamais eu de femme [...] pourquoi, au catéchisme, le curé dit toujours: Le Bon Dieu et sa grande clémence?» ( $M R$, p. 312), etc.

Parallèlement à l'inscription du double comme fondement du jeu verbal chez Devos, il faut noter les effets de miroir tout aussi prépondérants dans les textes, sur le plan de la structure narrative. Une phrase dans L'artiste $(M R, \mathrm{p} .19)$, Le vent de la révolte $(M R$, 
p. 193), Le clou ( $M R$, p. 374) ou Le pied ( $M R$, p. 388), un paragraphe entier dans La quatrième dimension (MR, p. 123), Le petit poussin ( $M R$, p. 83) et La porte ( $M R$, p. 24) sont répétés à plusieurs reprises à l'intérieur du monologue; parfois même, c'est un épisode intégral de l'histoire qui est repris, comme dans Les oublis ( $M R$, p. 379). Tout un texte, par ailleurs, est construit sur ce type de double, illustrant du même coup le principe de la mise en abyme. Au long du monologue Qu'est-ce qui vous arrive!, en effet, chaque fois que Devos amorce un nouvel épisode de son récit, il se rapetisse de quelques centimètres et diminue la portée de sa voix jusqu'à devenir inaudible, jusqu'à s'allonger par terre:

Tout à l'heure, en arrivant ici, je croise un type... il était petit comme ça... [...] la veille, je l'avais rencontré... Il était comme ça (il reprend sa taille normale). Et là, je le vois... Il était comme ça! (Il se rapetisse à nouveau). Je lui dis: - Qu'est-ce qui vous arrive? Il me dit: - Ne m'en parlez pas! Tout à l'heure, en arrivant ici, je croise un type... il était comme ça! (L'artiste se rapetisse de quelques centimètres de plus.) La veille, je l'avais rencontré... il était comme ça! [...] Je lui dis: Qu'est-ce qui vous arrive? Il me dit: Ne m'en parlez pas! Je viens de croiser un type... [etc.] ( $M R$, p. 52-53)

Le relevé des marques formelles de cette problématique du double serait incomplet si l'on omettait de noter une réduplication au niveau de la structure globale des spectacles de Devos. Quelquefois, c'est la reprise d'un même jeu de mots, en dehors du texte qui l'a vu naître, qui crée l'effet de miroir entre plusieurs monologues. C'est ainsi que la phrase "Un homme qui a chu n'est pas déchu..." dans Le savoir choir ( $M R$, p. 140) fait écho à "un ange qui a chu est déchu!!" dans Un ange passe, (MR, p. 101), et que "Qu'est-ce qu'il est encore allé brandir ailleurs?" ( $M R$, p. 197) dans Le vent de la révolte semble répondre à "un homme qui ne brandit plus, il est hébété. Quelquefois, j'entends sa femme qui lui crie: - Mais brandis autre chose!" (MR, p. 77) dans Le clairon. À d'autres occasions, c'est l'intervention d'un même personnage au sein de différents récits qui alimente le processus de duplication. Le personnage de Ceinture de sécurité réapparaît ainsi dans Tours de clefs ("Obligé de faire les trente kilomètres [...] en redoutant de rencontrer le type avec sa voiture en bandoulière!", $M R$, p. 42) et dans Supporter l'imaginaire ("C'est moi qui porte ma voiture en bandoulière!», $M R, \mathrm{p}$. 111). Un procédé équivalent ramène dans différents textes l'ange, le petit poussin et le primitif. Finalement, signalons dans la même veine les situations similaires de $L a$ 
106

quatrième dimension et de L'apparition de la parente, ou encore l'allusion dans des monologues ultérieurs à des textes donnés précédemment dans le spectacle. Les gens sont très marqués par ce qu'ils font ( $M R$, p. 378) et Le clou ( $M R$, p. 374) sont de fait rappelés - prodigieux paradoxe! - dans Les oublis ( $M R$, p. 381), comme Le rire primitif ( $M R, \mathrm{p} .65)$ dans Il y a quelqu'un derrière ( $M R$, p. 106) et dans Les objets inanimés (MR, p. 160).

Face aux procédés rhétoriques et narratifs utilisés par Raymond Devos, il nous revient, à présent, de souligner la cohérence remarquable de son œuvre. Il ne s'agit pas, comme c'est souvent le cas chez la plupart de ses camarades de scène, d'élaborer simplement une transition entre la fin d'un monologue et le début du suivant. C'est davantage une construction langagière et narrative parfaitement rigoureuse que Devos nous donne à apprécier, à la mesure des plus grands textes littéraires. Dès lors, il n'est pas inutile de se souvenir que Devos fonctionne sur le principe d'un même spectacle depuis pratiquement ses débuts sur scène, un certain nombre de textes récents (mais aussi de moments musicaux ou acrobatiques) étant substitués chaque fois à d'autres plus anciens. Quoi qu'il en soit, la présence du double, déclencheur ou conséquence des effets de miroir incessants entre les différents textes et fondement privilégié de la composition des jeux langagiers, s'accroît avec les derniers textes comme pour attester la maîtrise toujours plus grande de son art. L'écriture réfléchissant sur elle-même toujours davantage, l'architecture des textes et des spectacles se fait plus complexe, et les glissements ininterrompus du narrateur au personnage et à l'artiste lui permettent de se refléter lui-même à l'infini à travers les doubles qui naviguent dans Matière à rire ${ }^{3}$.

\section{Raymond Devos, poète de l'imaginaire}

Cela étant, le texte n'hésite pas à retourner sur le langage sa propre étrangeté et celle de certaines de ses formes pour le moins bigarrées: "Il y a des expressions curieuses" ( $M R, \mathrm{p} .286)$; "Il y a des verbes qui se conjuguent très irrégulièrement" $(M R$, p. 81); "Mesdames et messieurs, si vous voulez bien me prêter une oreille attentive... Quelle phrase! [...] Il y a des phrases

3 Voir à ce propos Michèle Nevert, Devos à double titre, Paris, PUF, 1994. 
comme ça..." ( $M R$, p. 79). De cette manière, Devos se décharge sur la langue de la responsabilité de quelques-unes de ses productions, les plus extravagantes comme les plus réussies. Ainsi, formes arbitraires et illogiques de la langue, effets intraduisibles du français s'imposeraient à l'auteur qui se contenterait seulement de les signaler? Devos serait un auteur docile et soumis à la langue, à la limite même de la passivité? On en doute et on penserait plutôt ici à l'ambivalence d'un artiste partagé entre l'humilité de reporter sur son objet de travail sa propre création et son besoin (d'autant plus essentiel par contrecoup) d'affirmer la réalité de son travail créateur. Ambivalence aussi par rapport à la valeur artistique de la création obtenue, puisque tout en persévérant à élaborer son œuvre, il lui arrive de minimiser parfois l'art qui est le sien. Ainsi dans $L e$ besoin d'ânerie: "on commence par dire des âneries... Ensuite, on sort quelques balourdises... Puis des stupidités, et de stupidités en stupidités... on en arrive aux inepties et, un jour, on se surprend à proférer des énormités" ( $M R$, p. 365). Le fait que, à l'entendre, il ne puisse faire autrement - "Chez moi, c'est un besoin! [...] C'est une mauvaise habitude que j'ai prise. [...] Si je reste dix minutes sans émettre une ânerie, je suis malade!"--, nous laisse présumer que les âneries en question pourraient bien renvoyer aux jeux de langage et que, pour leur part, les "énormités" concernent l'univers imaginaire qu'il crée. Dans Tours de clefs, le conteur achève son histoire en dénonçant justement l'exagération de ses propos: "C'est tellement énorme... que... je ne l'ai pas cru! ( $M R$, p. 43) C'est que l'escalade incontrôlable des "âneries" aux "énormités" débouche sur une conclusion inévitable: "Il est trop tard, l'esprit est faussé!" (MR, p. 365), avec ses conséquences malheureuses: "Attention! Vous allez empoisonner l'esprit de ceux qui vous entourent!" $(M R$, p. 365). Double danger pour l'homme qui joue avec les mots: se perdre soi-même à l'intérieur de la folie du langage, et entraîner avec lui le spectateur ou le lecteur. Mais si les mots échappent au contrôle de l'écrivain, ne pourraient-ils pas alors se rebeller contre leur auteur (voire leur lecteur)? Voilà justement le drame du schizophrène terrassé par la révolte des mots "stupéfaits de leur sort " - pour citer un autre poète ${ }^{4}$. L'artiste énonce à propos

4 "J'entends la plainte de chacune de leurs pages; le cri des vocables stupéfaits de leur sort ", Edmond Jabès, L'ineffaçable. L'inaperçu (Le livre des ressemblances, $I I)$, Paris, Gallimard, 1980, p. 9. 
des jeux de langage les mêmes mises en garde et recommandations que celles qu'il adresse à son public au sujet de l'imaginaire: "lorsqu'on a la prétention comme moi, d'entraîner les gens dans l'imaginaire, il faut pouvoir les ramener dans le réel, ensuite... et sans dommage!" ( $M R$, p. 108$)$; "il ne faut jamais lâcher dans la nature des papillons qui n'existent pas! Ça crée des fantasmes! [...] Il faut dissiper tout ça!» (MR, p. 303). Cette équivalence établie entre le langage et l'imaginaire ne surprend pas, si l'on songe que ce dernier, chez Devos, s'élabore essentiellement à travers le langage et grâce aux torsions qu'il lui fait subir. N'est-ce pas justement ce que suggère le monologue La boule volante: "il y a des magiciens qui vous promettent la lune... Moi, je vous promets le soleil... rien que par la puissance de mon souffle! " $(M R$, p. 33)? Car derrière le gag verbal, production inévitable de l'humoriste, c'est peut-être l'étrangeté et l'interrogation qu'il suscite qui importent le plus: "Il y a des choses bizarres!... il y a des choses inexplicables... des choses qui vous échappent!... L'autre jour, au café... je commande un demi... j'en bois la moitié!... Il n'en restait plus!» $(M R$, p. 434) Tout se passe comme si le jeu de mot, au départ, plutôt une fin en soi, se réduisait au bout du compte à un moyen, à un outil pour rompre avec le réel. La polysémie dont souffrent ou rient certains vocables, est, chez Devos, une clé, un passeport, une sorte de Sésame ouvretoi. Il prend le mot à contre-pied, relittéralise une expression figée ou encore recourt à tout autre jeu verbal (calembour, contrepèterie, etc) et, aussitôt, le réel bascule dans l'imaginaire. Sans minimiser l'apport fondamental de la manipulation langagière, on constate, malgré tout, que cette manipulation, qui demeure une caractéristique majeure de l'œuvre, est, bien plus qu'une performance verbale valant pour elle-même, le moyen de pénétrer et de traverser un univers. Conduit donc par le jeu verbal, l'univers de Raymond Devos est avant tout celui de l'imaginaire. Et d'ailleurs, au fur et à mesure de l'élaboration de cette œuvre, l'imaginaire occupe une place de plus en plus prépondérante. Si le jeu verbal conserve son statut privilégié, il apparaît, cependant, que sa fonction consiste à déclencher et à maintenir l'univers onirique.

\section{Raymond Devos, poète et psychanalyste}

Parce que Matière à rire traite de l'imaginaire, parce que des questions qui concernent l'identité et le corps, voire la sexualité, 
traversent ses textes, parce que ceux-ci sont construits autour d'un jeu incessant sur le signifiant, l'œuvre de Raymond Devos interpelle la psychanalyse et entretient avec elle un véritable dialogue. "Il y a des choses inexplicables... des choses qui vous échappent !..." écrit-il ( $M R$, p. 434). Mais qu'est-ce à dire? Qu'elles restent hors de portée, ou qu'elles sortent de vous malgré vous? Les deux, bien sûr. Si elles demeurent sans explication, si elles restent inintelligibles, ces "choses" encore sans nom, c'est parce qu' elles sont sorties de nous sans notre accord. Le plus souvent même à notre insu. Si, dans la même veine, il y en a "qui disparaissent et dont personne ne parle" ( $M R$, p. 235), c'est justement parce que ces choses, refoulées, ont quitté la conscience. Elles feront retour, cependant, sous d'autres formes, dans des formations de compromis, et plus difficilement compréhensibles pour avoir été soumises aux exigences de la censure qui veille sur notre sérénité de surface.

Une phrase des Ombres d'antan pourrait nous laisser croire que l'artiste dénie tout intérêt à tenter de découvrir ce qui se cache sous la surface: "On ne sait plus ce que c'est que l'obscurité. À force de vouloir faire la lumière sur tout, on ne distingue plus rien!" ( $M R$, p. 116) Il n'en va pourtant pas ainsi, puisqu'en poursuivant son élan, le texte fait état de l'avantage qu'il y a à déplacer les éclairages, à changer le point de vue de l'observateur: "je croyais connaitre cet endroit... Eh bien, à la faveur de la pénombre, j'y découvre des choses..." ( $M R$, p. 116).

\section{Surmoi et castration}

Mais qu'est-ce que l'artiste a bien pu déceler grâce à ce nouvel angle de vue et qu'est-ce qu'il cherche à nous indiquer? Car l'artiste dans le même élan occupe la place de l'analysant sur le divan et celle de l'analyste dans le fauteuil (de la même manière qu'il sait fort bien, linguiste à son insu, expliciter les jeux de langage qui engendrent ses textes). Dans un premier temps, les fantasmes habituels qui entourent l'organe sexuel masculin: "Il a sorti un sécateur et clac! (dans le vif du sujet). [...] J'en ai eu le souffle coupé... J'avais perdu de ma superbe. Savez-vous comment a fini mon beau grain dé raisin? Piétiné... écrasé sous les pieds d'un dénommé Bacchus [...]" ( $M R$, p. 14). Ce que la psychanalyse nomme castration, c'est en réalité la punition ultime dont est menacé métaphoriquement le spectateur du Cavalier et 
sa monture. Rappelons-nous comment celui-ci retourne chez lui, et avec deux réelles amputations: "[...] le spectateur est rentré chez lui en cavalier manchot sur cheval unijambiste!" ( $M R$, p. 153). Les mises en garde que Devos formule à l'égard de la castration sont nombreuses dans Matière à rire, quoique l'avertissement donné ici soit déjà largement suffisant par luimême; mais avec cette insistance, l'auteur ne fait qu'imiter la dynamique de l'inconscient qui ne cesse de se répéter pour s'assurer d'être un jour entendu à travers ses nombreuses variations.

Aussi les textes multiplient-ils les coupures à différents niveaux. À l'occasion, il s'agit du narrateur, l'artiste étant toujours le premier à endosser et à assumer les difficultés. On peut se référer bien entendu au Pied de vigne ou l'imagination de la matière déjà cité: "Vous n'aviez rien à cacher? Certes! Mais je n'avais tien à montrer non plus!» $(M R$, p. 12). Mais cela se traduit de manière plus voilée, dans La vie, je me la dois: "je me suis retrouvé au pied de l'escalier avec une jambe cassée." ( $M R$, p. 206) Ailleurs, c'est une femme qui fait les frais de la castration, subtilement incluse dans l'ambiguité du référent d'un pronom: "je viens de croiser une dame [...] Je l'enlace! Elle était comme ça! (il indique l'extrême minceur de la taille.) La veille, je l'avais enlacée... Elle êtait comme ça! (Il indique l'extrême grosseur de la taille) [...] Elle me dit: - Hier, ce n'était pas moi! Je l'ai coupé aussi!" $(M R$, p. 55). Le pronom "l'" renvoie-t-il au chapitre qui rapporte l'anecdote comme l'implique l'accord du participe passé ("coupé") ou, malgré la forme fautive pour l'œil ("coupée"), à la femme en question? Aux deux vraisemblablement. Car si dans Qu'est-ce qui vous arrive? c'est le texte qui subit la punition: "j'ai fait des coupures! C'est la version raccourcie. [...] j'ai coupé un chapitre. (MR, p. 55), le monologue Les objets inanimés tend à nous laisser croire, cette fois, que c'est du personnage qu'il s'agit: "C'est ainsi qu'elle est devenue ma chose! Mais... les choses n'étant que ce qu'elles sont, depuis [...] mes désirs sont sans objet [...]" (MR, p. 165) Le texte donc, l'homme, la femme, tout est susceptible de vivre une castration dans Matière à rire puisque l'essentiel est d'y faire passer un vrai message qui soit un message de vérité. C'est pourquoi le narrateur reconnaît aisément, dans Le vent de la révolte, avoir "tranché arbitrairement!" (MR, p. 194)

Devant la menace de telles représailles, le psychanalyste reconnaîtrait la présence et l'action d'une force supérieure, 
autoritaire, exigeante, peu encline à la compassion: le Surmoi, sans aucun doute! Les visages que prend ce dernier sont pour l'essentiel au nombre de deux dans l'œuvre de Devos: le gendarme (ou le policier) et Dieu. L'image d'autorité absolue que représente le divin n'attend pas de gloses particulières. Un seul exemple même suffit à étayer cette thèse: "Où se cacher? [...] il y a Dieu là-haut qui voit tout..." ( $M R$, p. 91) Quant au gendarme, il est l'incarnation idéale de la loi et de l'interdiction de la transgresser. Le policier apparaît d'autant plus répressif que même si l'artiste a chaussé des lorgnons qui lui donnent la perception singulière d'un monde systématiquement réduit ("Derrière mes verres, je vois tout petit!n, $M R$, p. 44), il reste néanmoins "immense", "avec un grand képi de travers" et "deux gros yeux..." (MR, p. 45) Mais non content de se prévaloir d'une taille gigantesque et d'un regard scrutateur lui aussi omniprésent, cette figure d'autorité si fréquente dans les textes vient doubler ${ }^{5}$ l'artiste sur son propre terrain: "Que vous soyez dans l'imaginaire, c'est normal, vous, vous êtes l'artiste! Mais moi, je suis gendarme et je ne suis pas dupe!n $(M R, \mathrm{p} .113)$. Le malaise qui s'ensuit chez le narrateur d'être ainsi toujours observé, "jugé ", y compris même dans son imaginaire, se traduit par les sentiments de culpabilité déjà décelés. Il se dévoile encore dans des inquiétudes mal fondées: "Cela m'arrive parfois quand je suis tout seul. Tout à coup, j'ai l'impression qu'il y a quelqu'un derrière moi." ( $M R$, p. 104) Comme si une faute épouvantable poursuivait le texte et présidait sans cesse à son écriture... Cependant, le représentant du Surmoi doit parfois perdre lui aussi un peu de sa superbe; il lui arrive d'être ridiculisé ou tout simplement récusé : "- Police! — Je ne vous crois pas [...] - J'ai l'uniforme! — Justement, [...] ça fait déguisé !" (MR, p. 427) Le désir de diminuer le poids de cette instance intraitable peut amener un récit, par une inversion des rôles du gendarme et du voleur, à transformer le policier en victime et le délinquant en défenseur de la loi : "Monsieur l'Agent, je viens d'entendre quelqu'un crier au voleur, au voleur! - Je sais, c'est moi! On vient de me voler ma montre." (MR, p. 532) Ce qui permet à l'instance de surveillance d'assouplir son caractère intraitable et de reprendre sans trop de trouble sa fonction initiale de protection du sujet contre lui-même. Les

5 "Non mais, qui c'est l'artiste?! " (MR, p. 216), "C'est moi qui ai entraîné ce spectateur dans l'imaginaire (MR, p. 113); "Moi, je ne suis pas dupe, je suis l'artiste! : (MR, p. 109) 
112

choses retrouvent leur aspect rassurant: "J'ai jeté un coup d'œil dans la rue... Sur le trottoir d'en face... il y avait un agent qui faisait les cent pas... Eh bien!... ça m'a rassuré!” ( $M R$, p. 416) Dualité inhérente à l'être humain partagé entre son désir et la peur ou le remords que suscite la jouissance: "J'ai zappé avec une telle rapidité que les images [...] se sont superposées. Quand j'ai vu le visage de Thérèse sur le corps d'Emmanuelle, j'ai dit oh! oh! où tu vas là? Si Thérèse te trouble à ce point, reste sur Emmanuelle!. ( $M R$, p. 175)

Devant une telle démonstration du rôle et du fonctionnement du Surmoi dans l'organisation psychique, on ne s'étonne pas que l'artiste mentionne l'état de tension permanent qu'éprouve le Sujet en butte à son inconscient: "[...] c'est terrible d'avoir ad vitam ceternam quelqu'un derrière soi qui vous pousse à faire quelque chose alors que l'on sait pertinemment qu'in extremis, il vous empêchera de le faire." ( $M R$, p. 360). Ainsi, et à défaut d'un Surmoi suffisamment solide pour assumer le contrôle des pulsions, la castration symbolique offre une solution immédiate à l'angoisse qui fait problème. La menace demeure dans le texte comme un avertissement à ne jamais oublier. Mais, grâce à cette présence à la fois inquiétante et structurante du Surmoi, une évolution peut se faire jour et diriger le gros de l'ouvre vers un épanouissement. Le texte affirme dans ses débuts n'avoir "rien à montrer" $(M R$, p. 12) ou être encore beaucoup trop "jeune pour jouer à ce jeu là " (celui du "papa et de la maman ", $M R$, p. 18). Et pour cause: le développement de l'individu n'implique-t-il pas toujours au départ une incertitude quant à l'identité sexuelle, avec toutes les inquiétudes et les désapprobations qui peuvent l'accompagner?: "la vilaine fleur! Mi-figue, mi-raisin!... Bisexuée! De nos jours, la bisexualité chez les fleurs, personne n'y trouve à redire... Mais à l'époque, être à la fois la fleur de la femelle et la fleur du mâle, c'était plutôt mal accueilli!» ( $M R$, p. 13) Il reste cependant que le parcours habituel de l'être humain le conduit, "à partir d'un désir cent fois caressé ", à faire naître charnellement "un beau grain de raisin..." (MR, p. 13).

En parallèle, et selon un parcours analogue, la poursuite ininterrompue de l'écriture conduit des sketches isolés et très courts à une ouvre cohérente, parfaitement élaborẻe, accomplie. Et, de fait, à travers certains monologues qui par moment font penser à de purs exercices de style (Ouï dire, Le pot de grès, Je hais les haies, etc.) comme par le biais d'autres textes davantage 
tendus vers une écriture indéniablement poétique, Devos élève le jeu de langage au niveau de la littérature, continuant ainsi l'entreprise déjà amorcée par plusieurs écrivains.

\section{La question du texte}

$\grave{A}$ un tout autre niveau, Matière à rire questionne également la psychanalyse dans le rapport originel qu'elle entretient avec la lecture des textes littéraires. Depuis les travaux déjà anciens de Charles Mauron ${ }^{6}$ et ceux, plus récents, de Jean Bellemin-Noël ${ }^{7}$, entre autres, le rapprochement entre littérature et psychanalyse divise les tenants de l'auteur dans son texte et ceux qui se désintéressent délibérement du premier pour s'en tenir au texte et au rapport du lecteur au texte. Partant, certains font avant tout de l'exprimé et du contenu l'objet de leur analyse, quand les autres exploitent davantage l'expression et le travail du texte. Passant outre à la divergence des questions posées par ces différentes écoles - s'agit-il de l'inconscient de l'auteur dans le texte? du travail inconscient de l'écriture, du texte même? -, Matière à rire ouvre une autre perspective, une perspective de convergence, qui oblige le lecteur à lui poser tour à tour ces diverses questions. Le travail particulier de l'écriture est irréfutable dans tous les textes, ces exercices de style qui sont autant de preuves vivantes de la réalité mentale de ce que la théorie psychanalytique nomme associations. De même, l'interrogation de l'auteur sur son identité. Tout cela transparait dans l'ouvre entier, par l'intermédiaire de réseaux associatifs consciemment déterminés, cela va sans dire, mais qui fonctionnent exactement sur le modèle des réseaux que tissent les forces inconscientes.

La linéarité et la logique du discours soumis à l'espace-temps coutumier sont pour la psychanalyse des traits secondaires par rapport à une autre cohérence plus enfouie, plus profonde, que le psychanalyste cherche précisément à mettre au jour dans le travail en commun avec l'analysant. C'est aussi le sens de l'indication donnée par Matière à rire quand le livre fait fi de la chronologie réelle de la rédaction des différents textes. Les périodes

6 Charles Mauron, Des métapbores obsédantes au mythe personnel. Introduction à la psychocritique (Baudelaire, Nerval, Mallarmé, Valéry, Corneille, Molière), Paris, José Corti, 1963.

7 Jean Bellemin-Noël, Vers l'inconscient du texte, Paris, PUF, coll. "Écriture ", 1979 , p. 203. 
114

délimitées dans cette édition intégrale de l'œuvre sont très vagues et inégalement réparties dans le temps: quatorze ans pour la "première période" - paradoxalement la plus récente - , sept ans pour la seconde, douze ans pour la troisième, deux ans pour la quatrième, ultime période donnée dans le texte mais, en réalité, première dans l'œuvre de Raymond Devos.

La lecture psychanalytique "fait répondre le texte aux questions qu'il nous pose à nous " 8 et qu'il se pose à lui-même. Le caractère polyphonique du signe permet au texte, "à la parole", de signifier tout autre chose que ce qu'elle dit. Cette "autre chose" à quoi fait peut-être référence le texte inédit intitulé Le chercbeur: "Je cherche ce que les autres n'ont pas trouvé [...]. Là où les autres n'ont pas cherché!"9 Les psychanalystes nous disent que cette "autre chose" voilée, cachée, déplacée se fait entendre "entre les lignes", quelquefois, ou de préférence par le seul jeu du "signifiant". "Je hais les haies qui nous emmurent" (MR, p. 363) dit Devos qui, merveilleux psychanalyste, précise aussitôt: "Je hais les murs qui sont en nous!". Le message poétique se double ainsi d'une autre dimension de sens qui relève pour sa part de l'inconscient et se constitue essentiellement en jouant sur les sons. Or, ce jeu sur le côté signifiant des signes est bien l'objet même de l'entreprise de Devos. Dans le monologue Le pot de grès, le texte indique clairement que ce n'est pas l'objet en tant que tel qui fait problème - un simple pot mais la matière même dont il est fait: " $\grave{A}$ dire vrai, ce n'est pas le pot qui n'agrée pas, c'est le grès! [...] Malgré que ce grès m'agrée, je vous saurais gré d'échanger ce grès contre un autre pot plus à son gré." ( $M R$, p. 234) Bien des analystes ne se priveraient pas de relever ici le procédé métonymique, un procédé courant du langage de l'inconscient, qui conduit le texte à parler d'un contenant, en lieu et place du contenu - à parler du pot plutôt que de ce qu'il contient. D'autant que l'insistance pour exprimer l'importance de la matière met d'elle-même le lecteur-analyste sur la piste de l'excrément, premier produit tangible de l'enfant, auquel ce dernier accorde le prix que cela mérite!

Ainsi, un écrivain, un de plus, met en lumière la prévalence du matériau de l'écriture en tant que tel, reléguant au second

8 Jacques Lacan, Ecrits, Paris, Seuil, 1970-1971, p.381.

9 Raymond Devos, Le chercheur (inédit), janvier 1977, 3 p. (Communication personnelle). 
plan le contenu et l'intention. Il faut dire que lorsqu'il est compris au sens de moyen de communication, le langage est plus souvent qu'à son tour fauteur de troubles, Devos le sait mieux que personne: "si je tire la langue [...] c'est compris de tous! Parce qu'une langue, c'est une langue! C'est la même pour tout le monde! Alors que si vous parlez la vôtré, on dit: - Qu'est-ce qu'il dit?" ( $M R$, p. 70) Le corps, dans cette perspective, redevient un moyen de communiquer plus direct, plus efficace peut-être que le langage lui-même. C'est que, trop souvent, soit "la pensée est mal émise ou elle est mal reçue", soit "c'est l'esprit de votre correspondant qui est occupé", ou alors le vôtre "qui est en dérangement!" ( $M R$, p. 258) Quelles qu'en soient les raisons donc, la communication est mal établie et l'essentiel du message, on s'en doute, ne parvient pas à son destinataire. Il y a un danger et une frustration réelle à utiliser le langage, qui si souvent s'oppose à nos désirs ou se tient toujours du moins à côté d'eux. Ses tournures singulières nous prennent au piège de leurs limites: "On vous met devant un choix! "Faites l'amour, ne faites pas la guerre." Il y en a peut-être qui voudraient faire autre chose!" $(M R$, p. 246) Voilà pourquoi, parfois, "on arrive à demander le contraire de ce que l'on veut!» ( $M R$, p. 136). Mais peut-être pis encore, l'absence du langage a dans certaines circonstances plus d'impact que son emploi: "quand un homme ne dit rien alors que tout le monde parle, on n'entend plus que lui! Redoutable!" $(M R$, p. 281). De là, la souffrance inévitable que les mots euxmêmes vont engendrer: "J'ai mal aux deux "mots" à la fois." ( $M R$, p. 176) Une souffrance réelle, physique, puisque les mots et le corps vont jusqu'à se confondre: "pour la première fois, je souffre de ce foie-ci et de cette foi-là..." $(M R$, p. 176) et "à la fin, chacun ayant ravalé ses mots et bu ses propres paroles, il n'y eut plus que des éclats de "voie" digestive et des "mots" d'estomac!" ( $M R$, p. 87). On pense, à ce propos, à une remarque de Jean Bellemin-Noël disant que "le sens ne se lève que pour celui qui se met à "mâcher" la substance phonique" 10

La détresse éprouvée par l'humoriste est avouée, dans un premier temps, dans L'artiste: "l'artiste [...] est ballotté comme une bouteille à la mer, à l'intérieur de laquelle il y a un message de détresse. [...] la bouteille, c'est lui [...]" ( $M R$, p. 20), puis, une

10 Jean Bellemin-Noël, Le rire, conférence, Département d'études littéraires, Université du Québec à Montréal, 1986. 
116

seconde fois, dans le texte Musique caressante. Ce texte nous semble d'autant plus important qu'il est tronqué de sa dernière partie dans la publication de Matière à rire - indication de lecture dirait là-dessus Henri-Paul Jacques ${ }^{11}$ ! On se souvient que ce monologue a été joué - et remanié dans ce but par Devos dans le film de Godard, Pierrot le fou. En voici la partie la plus révélatrice, omise par la version reprise dans Matière à rire: "Alors cette musique que j'entends, elle n'existe pas peut-être? Cette musique qui me détruit! Cette musique qui m'a suivi toute ma vie! Cette tendresse, dedans... cet homme ne comprend pas. Vous m'entendez?" ( $M R$, p. 83). Dans le film, Devos achevait sa scène hors champ, renforçant ainsi l'idée qu'il ne parle qu'à luimême, ce qui rend encore plus troublante la suppression de ce passage pour la publication.

"Cet air", cette musique-là, le comédien avoue aussi avoir du mal à la supporter. Musique des mots, musique du langage très certainement, puisque c'est celle qui berce tous les textes de Devos. N'y aurait-il pas ici, alors, l'origine des différentes ambivalences qui parcourent l'œuvre de l'humoriste? Ambivalence devant la profession d'artiste, ambivalence face à l'humour, ambivalence face à l'imaginaire, ambivalence face à l'écriture. Un rapport ambigu relierait-il Raymond Devos au langage? À croire que l'écrivain qui aime plus que de raison le jeu verbal, éprouve pratiquement autant de "haine "pour l'objet avec lequel il entretient une relation d'amour depuis tant d'années. Du coup, on saisit mieux cette culpabilité qui accompagne parfois certains textes. Et c'est également pourquoi, devant le remords et la culpabilité ressentis à travers cette "haine" inconsciente, lorsque l'artiste déclare avoir voulu se "racheter", le prix à payer est si exorbitant: "je me suis payé un prix fou!" ( $M R, \mathrm{p} .64)$ Une dépense d'énergie affective que, au-delà de la dépense financière, tout analysant en cure, voire un lecteur analyste de textes, connaît fort bien!

\section{L'ambivalence face au langage}

Dans Le corps de l'ouvre ${ }^{12}$, Didier Anzieu explique notre ambivalence face au langage sur le modèle kleinien du bon et du

11 Henri-Paul Jacques, Du rêve au texte, Montréal, Guérin littérature, 1988, 348 p.

12 Didier Anzieu, Le corps de l'cuvre, Paris, Gallimard, 1981. 
mauvais objet. L'infans est sans cesse confronté à ses incapacités face au langage: il ne le parle pas, il ne le comprend guère. Cette situation implique une grande frustration et autant d'agressivité. Aussi, plus tard, l'enfant qui demeure en nous peut vouloir retourner contre le langage cette violence que le langage lui a, jadis, fait subir. Dans ce but, pour affirmer la maîtrise qu'il a acquise à présent, il manipule les mots comme des excréments, les décortique comme des aliments, les démonte comme des jouets, bref, il tente de déjouer les règles de la langue. Au cours de sa réflexion, Anzieu établit un lien évident entre le Surmoi et le jeu de langage:

Si l'on veut bien admettre, avec Freud, que le Surmoi a des racines acoustiques, et si l'on entend par là que le premier système complexe et cohérent de règles que l'entourage impose à l'enfant est celui de la langue [...], cela permet de comprendre pourquoi l'esprit $[\ldots]$ se sert de certaines règles, de certaines fonctions de la langue pour les mettre en contradiction avec d'autres règles, avec d'autres fonctions. ${ }^{13}$

Dans cette perspective, le désir destructeur qui se décèle dans les torsions imposées au langage "traduit la révolte du Moi contre le Surmoi en tant que celui-ci impose les règles de la langue avec un arbitraire qui fait violence " 14 . En revanche, lorsque coïncident harmonieusement la forme et le sens, "cette fusion des mots appris de la mère avec les parties du corps de celle-ci mêlées à d'autres parties du corps de l'enfant signe le triomphe du Moi idéal: je sais tout, je peux tout, je suis tout si les mots obéissent à mon bon plaisir ".

Chez Devos, ce désir de prendre le langage à ses propres pièges confine à l'extrême en même temps qu'il signe l'impossibilité, en bout de course, de l'abolir définitivement. À plusieurs reprises, l'artiste indique métaphoriquement l'incapacité ultime où on se trouve de s'extraire du cercle vicieux dans lequel le magicien des mots s'est laissé entraîner, et nous avec lui en acceptant de le suivre. Lisons Le plaisir des sens: "J'arrive sur une place... je prends le sens giratoire... [...] Je fais un tour pour vérifier. Quatre rues, quatre sens interdits !... [...] Alors? pour sortir?... Vous ne pouvez pas!„ ( $M R$, p. 474-475) Dans Ca n'a pas de sens: aje mettrais bien un béret; mais un béret non plus ça n'a

13 Ibid., p. 357.

14 Ibid., p. 343-344. 
pas de sens. Vous pouvez le tourner de tous les côtés, il est toujours de face." (MR, p. 421) Dans Sens dessus dessous: "je ne compte pas trop dessus $[. .$.$] parce que [...] au-dessus de celui qui$ est au-dessus, il n'y a plus d'appartement! Alors, le locataire du dessous qui monterait au-dessus obligerait celui du dessus à redescendre en dessous" ( $M R$, p. 222). Aussi, et si dans une volonté utopique de liberté absolue, le désir le plus fort est celui de se tenir en dehors des contraintes immuables du langage, les présentes comme les passées, l'unique solution qui reste offerte consiste à se mesurer au système en attendant qu'il "casse " définitivement de lui-même:

Je connaissais un sportif qui prétendait avoir plus de ressort que sa montre. Pour le prouver, il a fait la course contre sa montre. Il a remonté sa montre, il s'est mis à marcher en même temps qu'elle. Lorsque le ressort de la montre est arrivé à bout de course, la montre s'est arrêtée. Lui a continué, et il a prétendu avoir gagné en dernier ressort! ( $M R$, p. 245)

Une solution vouée forcément à l'échec puisqu'elle reste métaphorique, et donc toujours tributaire du système. Ainsi, à défaut de ne pouvoir réellement abolir le langage, on peut comprendre le désir de faire rire comme une manière d'opposer un nouveau refus de se soumettre au langage, ce code fondamentalement inébranlable. D'ailleurs l'envie de le détruire n'est pas proportionnellement plus grande que celle de le vénérer. Si cela devait être le désir premier du Sujet, celui-ci se tairait comme, du reste, certains psychotiques le font parfois.

Et faire rire, l'artiste y parvient - apparemment - sans difficultés. On le sait, on profite de cette immense liberté que s'offre en partage, et que nous donne en partage, Raymond Devos. Son talent signe le triomphe de son empire sur les mots, de notre éventuelle maîtrise absolue du langage. Une omnipotence toujours fantasmée et qui satisfait du même coup notre désir de rébellion. Car si les mots obéissent à notre bon vouloir, alors nous devenons capables de tout, détenant une puissance quasi cosmique. Et l'explosion finale qu'elle implique devient, en forme de leçon dernière, celle de Matière à rire, l'œuvre, mais celle aussi du texte qui porte le même intitulé:

[...] si l'on savait ce qui amuse les atomes, on leur fournirait matière à rire... Si bien qu'on ne les ferait plus éclater que de rire. Alors, me direz-vous, que deviendrait la fission nucléaire? Une explosion de joie! (MR, p. 297) 
Raymond Devos est un poète. Un homme qui a affaire aux mots. Il se bat avec eux et les caresse; les affronte et les adore, les aime. Il joue avec eux sur la scène, fascinant le public par sa toute-présence, mais il a d'abord écrit leurs aventures et mésaventures. Il les fait jouer, mis à nu ou déguisés, naîfs ou malicieux: enfants. Celui qui sait combien nos mots viennent de l'enfance, jusqu'où plonge leur enfance, comment cette enfance chante et déchante, celui-là est un poète... 